\title{
Novel Computational Methodologies for Structural Modeling of Spacious Ligand Binding Sites of G-Protein-Coupled Receptors: Development and Application to Human Leukotriene B4 Receptor
}

\author{
Yoko Ishino ${ }^{1}$ and Takanori Harada ${ }^{2}$ \\ ${ }^{1}$ Graduate School of Innovation \& Technology Management, Yamaguchi University, 2-16-1 Tokiwadai, Ube, Yamaguchi 755-8611, \\ Japan \\ ${ }^{2}$ Graduate School of Biomedical Sciences, Hiroshima University, 1-2-3 Kasumi, Minami-Ku, Hiroshima 734-8551, Japan
}

Correspondence should be addressed to Yoko Ishino, ishino.y@yamaguchi-u.ac.jp

Received 12 October 2012; Accepted 30 October 2012

Academic Editors: S. Jahandideh, P. Jain, and M. Liu

Copyright ( $) 2012$ Y. Ishino and T. Harada. This is an open access article distributed under the Creative Commons Attribution License, which permits unrestricted use, distribution, and reproduction in any medium, provided the original work is properly cited.

\begin{abstract}
This paper describes a novel method to predict the activated structures of G-protein-coupled receptors (GPCRs) with high accuracy, while aiming for the use of the predicted 3D structures in in silico virtual screening in the future. We propose a new method for modeling GPCR thermal fluctuations, where conformation changes of the proteins are modeled by combining fluctuations on multiple time scales. The core idea of the method is that a molecular dynamics simulation is used to calculate average 3D coordinates of all atoms of a GPCR protein against heat fluctuation on the picosecond or nanosecond time scale, and then evolutionary computation including receptor-ligand docking simulations functions to determine the rotation angle of each helix of a GPCR protein as a movement on a longer time scale. The method was validated using human leukotriene B4 receptor BLT1 as a sample GPCR. Our study demonstrated that the proposed method was able to derive the appropriate 3D structure of the active-state GPCR which docks with its agonists.
\end{abstract}

\section{Introduction}

G-protein-coupled receptors (GPCRs), the largest family of membrane proteins (around 800 in humans), are involved in a variety of biological and pathological processes such as development and proliferation [1], neurological disorders [2], angiogenesis [3], and metabolic disorders [4]. GPCRs transmit molecular information from the extracellular side to the intracellular side of the cell, thus mediating many intracellular responses. Although nearly one half of currently marketed drugs target GPCRs [5], only $10 \%$ of these receptors have endogenous ligands [6]. Structurally, all GPCRs share an architecture formed by seven transmembrane (TM) helices connected by extracellular and intracellular loops. GPCRs can recognize structurally diverse ligands ranging from photons to ions, amino acids, small organic molecules, lipids, peptides, or proteins [7].
Due to limitations in the purification and crystallization of GPCRs, until recently only a limited number of threedimensional (3D) structures of GPCRs have been resolved at high resolution by X-ray crystallography: rhodopsin [8], the $\beta$-adrenergic receptors $\left(\beta_{2} \mathrm{AR}[9,10], \beta_{1} \mathrm{AR}[11]\right)$, the adenosine $A_{2 A}$ receptor [12], and the opioid receptors $(\kappa$ [13], $\mu$ [14]). The highly conserved general architecture including the TM helical bundle and some universally conserved residues allows predicting the $3 \mathrm{D}$ structure of unknown GPCRs by homology modeling, while using a few known GPCR structures as a template [15]. However, great cautions are needed when utilizing the homologybased models for detailed GPCR structural and functional annotations since helix kinks are often different in different receptors. Modeling these subtle distinctions, which is essential for ligand docking and screening, remains a major challenge [16]. In addition, since almost all known GPCR 
structures have been found in their inactive forms, the homology modeling has shown little power to practically predict the $3 \mathrm{D}$ structure of GPCRs in the active forms. It is known that the agonist-bound GPCRs take the active forms, whereas the antagonist-bound or inverse agonistbound GPCRs take the inactive forms.

Ligand-induced activation of GPCRs results in multiple allosteric conformational changes that propagate throughout the receptor structure, ultimately triggering different signaling cascades [17]. In contrast to inactive states of GPCRs, no crystal structures of active states were available until 2011. Given the absence of experimental structural information, several investigators have applied computational strategies to predict activated models of GPCRs [18-22]. Therein, it is reported that applying an appropriate long-term molecular dynamics (MD) simulation after homology modeling could predict plausible 3D structures of activated GPCRs in view of the induced fit mechanism $[21,22]$. However, this approach needs to impose appropriate structural constraints prior to the MD simulation, which requires both the skills and experience of an MD specialist. Moreover, MD simulations on ordinary personal computers are generally limited to timescales up to the microsecond order in long-term studies, yet structural changes from fitting-induced fluctuations require calculations on the millisecond order. Due to its time-consuming nature, it is virtually impossible to simulate all processes involved in structural changes using only MD on common computers. For this reason, currently no highaccuracy methods are available for predicting the activated structure of GPCRs.

This paper describes a novel method to predict activated GPCR structures with high accuracy on standard workstations, while aiming for the use of the predicted $3 \mathrm{D}$ structures in in silico virtual screening in the future. First, we have developed a new method for modeling thermal fluctuations in GPCRs by using combinations of fluctuations with different time scales. Based on this model, we propose a novel computational method to search for one of the best $3 \mathrm{D}$ structures of an activated GPCR.

\section{Model and Framework}

Proteins undergo thermal fluctuations of considerable magnitude. The time scale of thermal fluctuations in proteins ranges from femtoseconds to minutes, or even longer timescales [23]. In terms of spatial considerations, various levels of fluctuations exist, which include rapid motions within microspace (such as thermal oscillations between atoms and rotations of amino acid side chains), and relatively slow but large structural changes (such as local unfolding). Not all of these motions can be simulated because of the physical limits of currently available MD calculations. We therefore propose a new method for modeling GPCR thermal fluctuations, in which fluctuations are combined on different time scales. In this approach, we first simulate the oscillations and fluctuations of various atoms at the picosecond and nanosecond levels to obtain native structure within a short time span. We then simulate the thermal fluctuations of GPCRs in terms of their characteristic rotational motions around helical axes, which represent fluctuations of greater time scales. In our approach, some information on GPCRs that is known a priori is utilized. For instance, we know that TM helices are immobilized in a cell membrane, so their mobility is certainly low. Furthermore, this approach is based on the previous findings that when a GPCR is activated in a cell membrane, its structural changes occur by rotations around the axes of TM helices [24], and that especially TM3, TM5, and TM6 play an important role in the activation process in some GPCRs $[18,21]$. Although this method does not guarantee the optimum from an exhaustive chemical calculation approach, it does have the clear advantage of reducing calculation load without sacrificing the quality of the solution obtained. We specifically focus on predicting the central part of the 7-TM helical bundle, a spacious ligand binding site, with good quality, while aiming for the use of the predicted $3 \mathrm{D}$ structures in in silico virtual screening in the future.

Based on this model, we propose a framework for structural search which comprises three stages, namely: (i) obtaining initial structures of a target GPCR by homology modeling using known GPCRs in their inactive forms as a template, (ii) obtaining average structures of a GPCR in a stable state after simulating fluctuations from several hundred picoseconds to a nanosecond using MD simulations, and (iii) obtaining a best 3D structure of a target GPCR in the active form through a machine-learning technique including evolutionary computation and modularized ligand-binding simulations. Our proposed method employs a real-coded genetic algorithm (GA) as an evolutionary computation to search appropriate rotation angles of helices of a target GPCR, in which discrete steps employ receptor-ligand docking simulations to evaluate the goodness of the rotation angles of the GPCR.

The core idea of the framework is that an MD simulation is used to calculate the $3 \mathrm{D}$ coordinates of all atoms of a target GPCR in a relaxed state in terms of heat fluctuation on the picosecond or nanosecond time scale, after which the evolutionary computation is used to determine the best ligand-bound rotation angles of helices of a GPCR as a movement on a longer time scale.

\section{Methodology to Predict 3D Structure of Activated GPCR}

The detailed procedure of the proposed structural search method is described as follows.

(1) Determination of an initial structure: a known GPCR such as rhodopsin or beta-adrenergic receptor is used as a template. After alignment of the primary amino acid sequences of the template and target GPCR, the inactivated structure of the target GPCR is generated by regular homology modeling, which results in an initial structure.

(2) Average molecular structures with respect to fluctuations on a short-time scale: an appropriate amount of water molecules are added to the surroundings of the 
target GPCR. The fluctuations of GPCR molecules are then calculated by MD simulations at the level of several hundred picoseconds. The average structures are defined as those for which the energy values stabilize.

(3) Structural changes with respect to rotational motions of helices: hypothetical rotational motions around the axes of helices constituting the target GPCR (as fluctuations of greater-time scale) are generated. These are fed into a real-coded GA [25], as explained above, including simulations of binding with known ligands to determine optimal values. Details of the steps are described as follows. In addition, the schemes for generation alternation and offspring creation are shown in Figure 1.

(3.1) Generation of initial populations. Individuals are assigned real-number vectors representing rotational angle changes relative to the average structures of various helices. These real-number vectors are treated as values within the space adjoining the two ends of the search space (i.e., toroidal space.) An initial population of $m$ individuals is randomly generated.

(3.2) Creation of offspring for each generation. Parents (two individuals) for random crossover of population pools are selected to match with a third parent, so as to generate offspring by the unimodal normal distribution crossover (UNDX) method [26]. The third parent is used to determine the standard deviation of normal distribution in UNDX. This procedure is repeated $n$ times to provide $n$ offspring individuals. In total, the number of individuals in a generation is $n+2$ (offspring and two parents).

(3.3) Selection in each generation. To evaluate each individual, receptor-ligand docking simulations are conducted using a known agonist and a known antagonist. An individual's real-valued vector translates into a corresponding $3 \mathrm{D}$ structure, with its side chains optimized before the binding simulations. An evaluation function has been established to represent how good the binding is. For a single individual, ligandGPCR docking simulations are conducted up to $k$ times, and each evaluation score is recorded. Upon obtaining the top score for each individual, the scores are then used to rank all individuals in a generation. The two individuals highest in ranking are selected (elite strategy) to replace the two parents in the population.

(3.4) Reiteration. Steps (3.2) and (3.3) are repeated until some stop criteria are met.

The values for $m$ and $n$ can be determined based on previous researches and/or preliminary experiments. In our proposed search method, generation transition is achieved

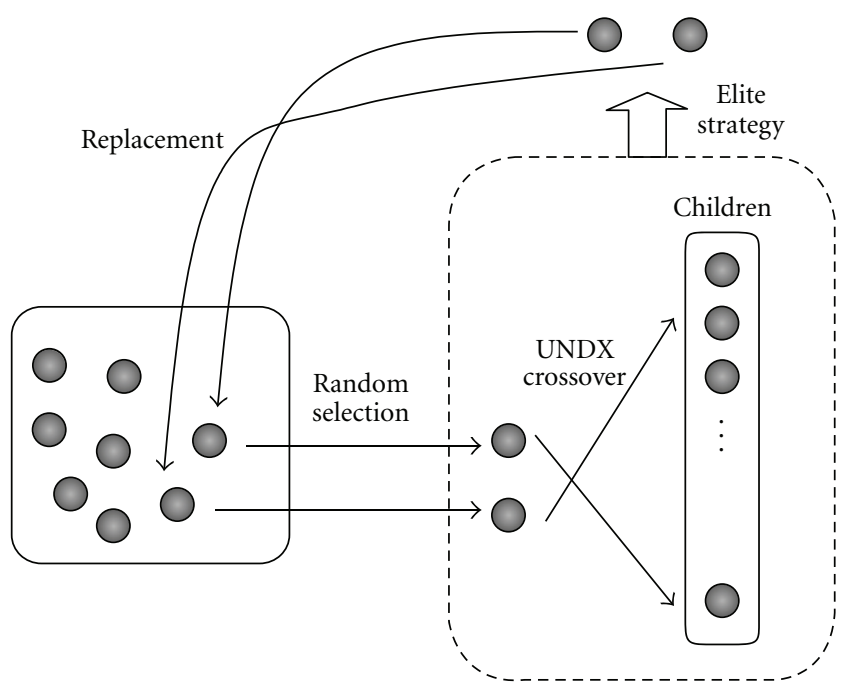

FIGURE 1: Schemes for generation alternation.

via the minimal generation gap (MGG) method [27]. MGG has good compatibility with UNDX and contributes to maintaining diversity in the population.

\section{Test Case of Human Leukotriene B4 Receptor BLT 1}

This study reports on a novel approach to molecular modeling of 7-TM proteins that has been developed to build plausible active states of any given GPCR by using evolutionary computation. This heuristic approach starts from the amino-acid sequence of a target GPCR. We performed a validation test, where human leukotriene B4 receptor BLT1 was used as a sample target GPCR.

4.1. Leukotriene Receptor. Leukotriene (LT) is a bioactive lipid that serves as an important mediator of host defense, though it is also known to be implicated in bronchial asthma as a pathogenetic or precipitating factor. To date, four types of LT receptors have been cloned. One of these receptors, the high-affinity human LTB4 receptor BLT1 (GPCRDB entry ID: LT4R1_HUMAN; UniProt entry ID: Q15722), was selected as a target GPCR in our experiments. The length of the amino-acid sequence of BLT1 is 352aa.

4.2. Experimental Procedure. The sequence of BLT1 was retrieved from the UniProt database. ClustalX software [28] was used to align the sequence with the crystal structure of the bovine rhodopsin (UniProt entry ID: P02699) with which it shares a sequence similarity of more than $54 \%$. One 3D structure of BLT1 obtained after running homology modeling using the bovine rhodopsin (PDB ID: 1L9H) as a template was selected from ModBase [29] as an initial rough $3 \mathrm{D}$ structure. ModBase is an open database of comparative protein structure models, theoretically calculated by the modeling pipeline ModPipe, which is maintained by Sali [30]. 
After annealing for atomic relaxation, MD simulations having distance restraints similar to a way proposed by Gouldson et al. [22] were run by using the TINKER software (ver. 4.2) [31]. The simulation conditions used were: force field $=$ AMBER99; temperature $=310 \mathrm{~K}$; pressure $=1 \mathrm{~atm}$; time step $=1.0 \mathrm{fs}$. An average structure was determined after reaching a stable state, at which no steep drop in the molecular energy was observed. MD simulations for several hundred picoseconds were needed to achieve such a stable state.

The following are the processes of an evolutionary computation method in terms of optimizing the rotation angles of TM helices. The previous work performing amino-acid residue substitution analysis and spectroscopic experiments found that "TM3, TM5, and TM6 play an important role in activation of the leukotriene receptor [32]." This let us bound the free rotation space searched to these three helices; hence, the solutions we seek are the rotation angles of the three helices. Our proposed method employs a real-coded GA as a search algorithm and the ligand-binding simulation as an evaluation tool. An individual, which is defined as a real number vector representing the rotational angles of TM3, TM5, and TM6, has a unique value. In the evolutionary search process, the genetic operations mentioned in the previous section are repeated until the point when the stop criteria are met (the maximum generation number is 200 , determined based on a preliminary experiment). The computational parameters in the evolutionary search are as follows: the initial population size is 50 , and the number of offspring in a generation is 8 . For evaluation of individuals, two sets of ligand-binding simulations are independently performed using a BLT1 agonist 12-keto-LTB4 (PubChem CID: 5280876) and a BLT1 antagonist pranlukast (PubChem CID: 115100). Docking of these ligands into the 3D structure of each individual, which is restored from the individual's vector representing the rotational angles of three helices to the 3D structure through structural relaxation by an ab initio (first-principle approach) computational method, is carried out with the GOLD software (ver. 3.1) [33]. The evaluation function in the evolutionary process is determined using the GOLD scores obtained from the docking simulations. The maximum GOLD scores derived from the docking with the BLT1 agonist and the BLT1 antagonist are assigned as $x_{1}$ and $x_{2}$, respectively. The evaluation function value is shown below.

When $x_{2}>0$, evaluation function value $=x_{1}-x_{2}$; for all cases other than this condition, evaluation function value $=$ $x_{1}$.

Finally, a best BLT1 structure obtained through the proposed method is evaluated by executing the docking simulations with many ligands other than ligands used in the evolutionary search process.

4.3. Results and Discussion. A computer with an Intel Xeon 3.6 GHz CPU (dual processor) was used for computation. For MD processing of 219.3-ps simulations, 524 hours were required. We investigated the changes in GPCR molecular energy values $(\mathrm{kcal} / \mathrm{mol})$ for every $0.1 \mathrm{ps}$ time elapsed in

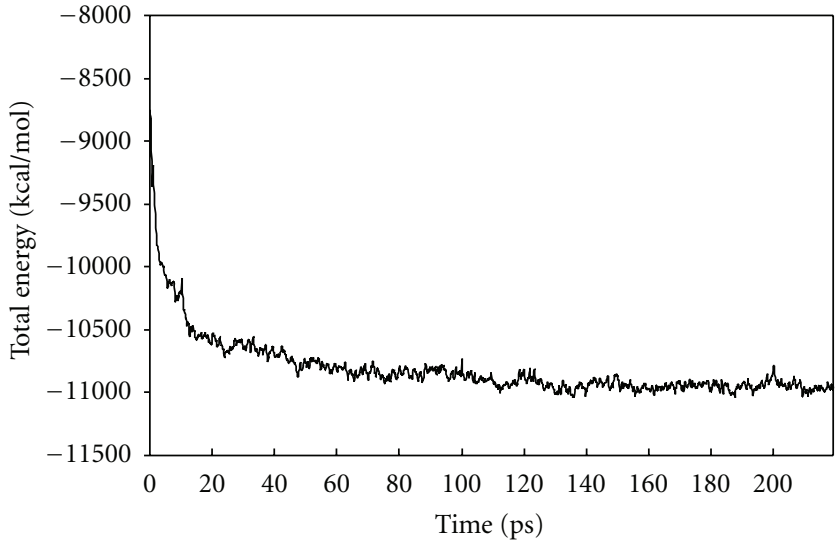

FIgURE 2: Molecular energy change of BLT1 through MD simulations.

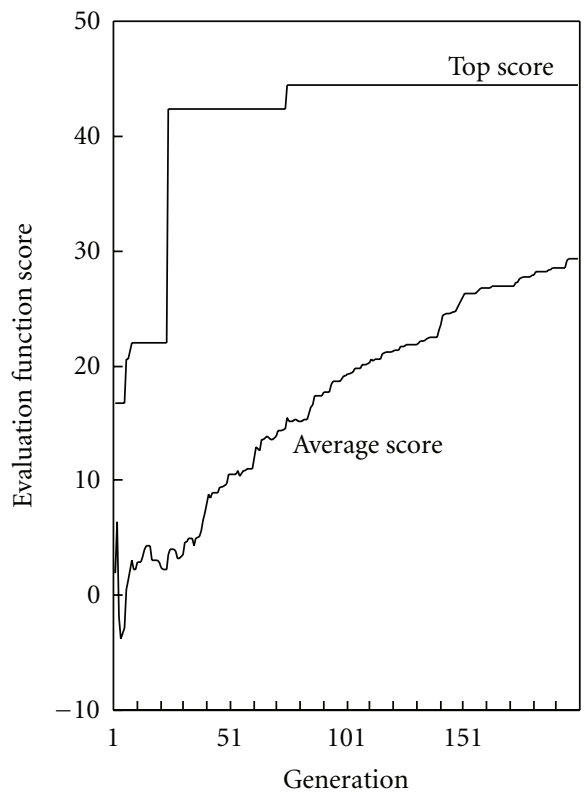

FIGURE 3: Evaluation function score in the evolutionary computation.

$\mathrm{MD}$, as shown in Figure 2. Lower-energy values suggest corresponding increases in structural stability. At the point of approximately $200 \mathrm{ps}$, energy values ceased to further fall, which suggests that a region of stability had been reached. As our study focused on MD simulations for structural changes on a short-time scale (meaning that the objectives were to simulate structural relaxation), we considered 200 ps to be a sufficient level and therefore stopped at $219.3 \mathrm{ps}$.

For subsequent evolutionary computation, 899 hours were required for calculations for 200 generations. Figure 3 shows the trends of scores in evolutionary computation (the higher the scores, the better). The optimal solutions for rotational angles after 200 generations were: TM3 $=11^{\circ}$, $\mathrm{TM} 5=14^{\circ}$, and $\mathrm{TM} 6=255^{\circ}$. 


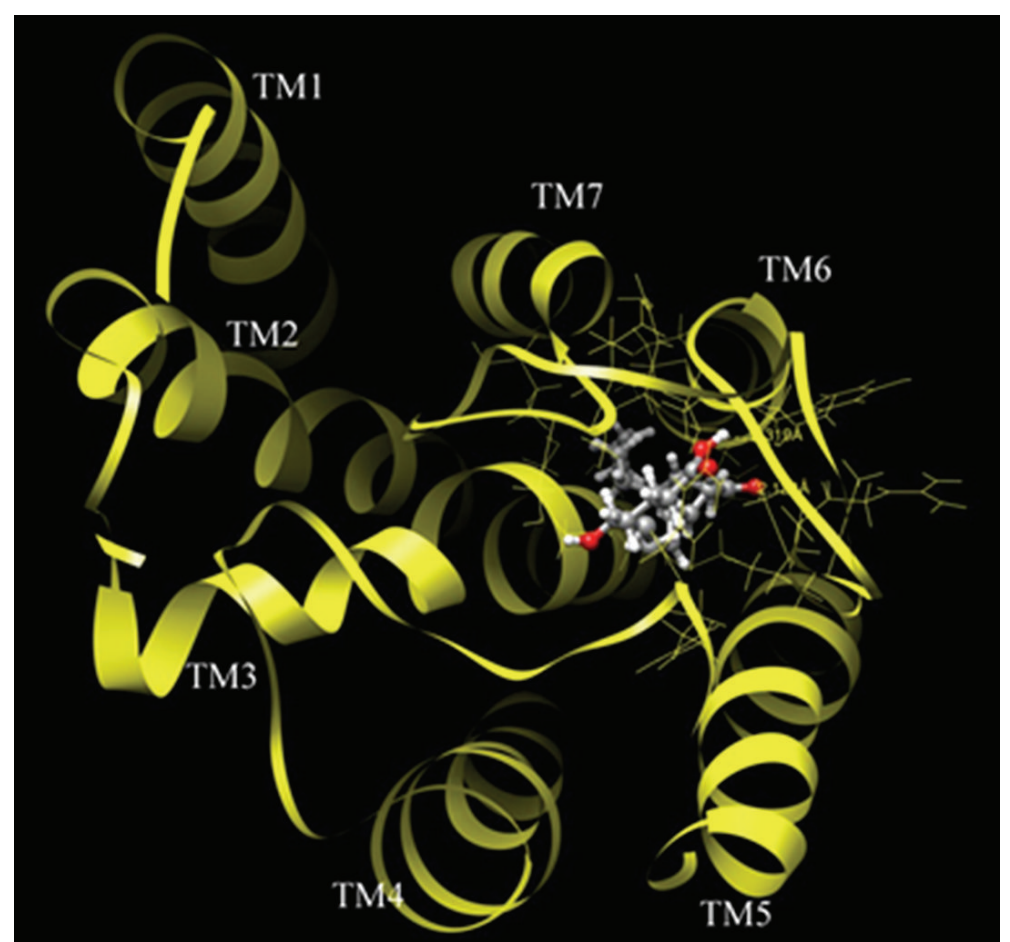

(a)

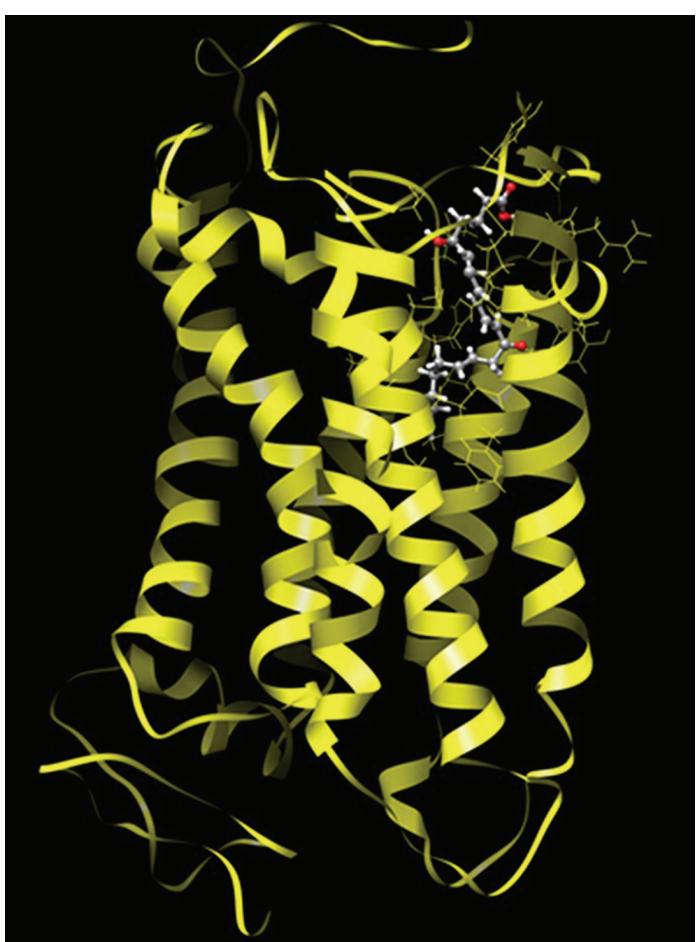

(b)

FIgure 4: A best 3D structure of active-state BLT1.

Figure 4 shows the state of binding between the LT receptor and its agonist in one of the best $3 \mathrm{D}$ structures computed. The 3D structures of binding are shown in Figure 4(a) with a view from above the cell plasma membrane and in Figure 4(b) with a cross-section of the membrane (the upper part being the extracellular space). In order to highlight receptor-ligand interactions, the main chains (backbones) of the helices are shown as ribbons, while the BLT1 agonist 12-keto-LTB4 is drawn as a ball-and-stick structure. From Figure 4, it is clear that the BLT1 agonist binds to a recessed region (pocket) formed by TM3, TM5, and TM6. Upon further analysis regarding the binding state of the LT receptor and its ligand, these helices responsible for interacting with the LT ligand are composed mostly of hydrophobic amino acid residues, suggesting that the BLT1 ligand is drawn into a strongly hydrophobic environment. Since the BLT1 agonist has many hydrophobic groups, it is likely that ligand-receptor binding is contributed to mainly by hydrophobic interactions, though several hydrogen bonding sites contribute to the binding. The following interactions are worth detailing.

(i) Hydrophobic interactions between TM3 and the alkyl side chain near the central carbonyl $(-\mathrm{C}=\mathrm{O})$ group of the BLT1 agonist.

(ii) Hydrophobic interactions between TM5 and the alkyl side chain near the central carbonyl group of the BLT1 agonist.

(iii) Hydrogen bonding between the $-\mathrm{OH}$ group in the carboxyl (-COOH) group of the BLT1 ligand and the carbonyl group of Asn241 on TM6 (C=O $\cdots \mathrm{H}-\mathrm{O}$ intermolecular distance $2.32 \AA$.).

(iv) Hydrogen bonding between the central carbonyl group of the BLT1 agonist and the main chain (-N$\mathrm{H}$ peptide bond) of Gly246 on the loop joining TM6 and TM7 $(\mathrm{C}=\mathrm{O} \cdots \mathrm{H}-\mathrm{O}$ intermolecular distance $2.13 \AA$ A.).

(v) Hydrophobic interactions between TM6 and all alkyl side chains of the BLT1 agonist.

In addition to TM3, TM5, and TM6, we speculate that TM7 also contributes to hydrophobic interactions with the alkyl side chains of the BLT1 agonist.

At the beginning of evaluation, we calculated the root mean square deviation (RMSD) of BLT1 structures to investigate the structure change. Firstly, we obtained initial structures from homology modeling as structure I, MD relaxed structures as structure II, and structures generated from post-MD evolutionary computation as structure III to calculate the RMSD of 1,316 atoms forming the backbones. The resultant RMSDs were $4.49 \AA$ for structures I and II, $1.55 \AA$ for structures II and III, and $4.63 \AA$ for structures I and III. Based on these values, the transition from structure I to structure II involved a large change. The reason why the transition from structure II to structure III was a smaller change is that the evolutionary search process only altered the rotation angle of TM3, TM5, and TM6. Nevertheless, what is interesting is that in the transition from structure II to structure III, the amount of rotational angle change in TM6 was clearly large compared with the other two TM 
helices: $\mathrm{TM} 3=11^{\circ}$, TM5 $=14^{\circ}$, and TM6 $=255^{\circ}$. This is consistent with other previous research showing that the TM6 region plays a very important role in the transition from inactive-state to active-state rhodopsin [34]. Since GPCRs including the leukotriene receptor are allosteric proteins, the conformational change from inactive to active state likely affects the state of engagement with the associated G-protein. However, details of this mechanism require additional research.

In order to check the validity of LT receptor structures obtained by our method, we further used 6 known BLT1 agonists (including 12-keto-LTB4) and 14 known BLT1 antagonists (including pranlukast) to independently perform binding simulations with BLT1 of the optimal 3D structure, from which the corresponding maximum GOLD scores were determined. The results are as shown in Figure 5 such that the agonists and antagonists are plotted in order of decreasing scores. The agonist group produced scores that were visibly higher than those of the antagonist group. By using the Wilcoxon signed rank test (which makes no assumptions on normality of distribution), the GOLD scores of the agonist group and the antagonist group were found to be significantly different $(P=0.00046)$. This strongly suggests that the binding pocket obtained by our search method selectively binds with the BLT1 agonists.

Next, in order to assess the quality of the agonist binding states, we have challenged the best $3 \mathrm{D}$ structure model with a set of 144 GPCR ligands to determine whether the structure indeed shows a good preference for BLT1 agonists. The ligand set included 6 BLT1 agonists (including 12-keto-LTB4), 14 BLT1 antagonists (including pranlukast), and 124 other GPCR ligands (32 against serotonin receptors, 17 against histamine receptors, 17 against adenosine receptors, 14 against prostanoid receptors, 10 against dopamine receptor, 8 against adrenoceptors, 8 against angiotensin receptors, 7 against cannabinoid receptors, and 11 against other GPCRs), as shown in Table 1. Being GPCR ligands, these ligands automatically have "drug-like" properties, so the only filtering on the ligand selection was to ensure that BLT1- and non-BLT1 ligands had a similar molecular mass distribution. The ligand-binding simulations were individually performed using the GOLD software. The overall docking results for the most highly ranked conformations are shown in Figure 6. Based on the GOLD binding score (higher indicates better binding), all 6 BLT1 agonists were ranked in the top 11. The enrichment curve in Figure 6 shows that there is immediate enrichment, and the results are good compared to related studies $[22,35]$. This also suggests that the binding pocket obtained by our search method selectively binds with the BLT1 agonists.

Consequently, we have developed a search method capable of predicting the 3D structures of active forms of GPCRs, which is broadly consistent with known facts of the target proteins and is reasonably persuasive as a model.

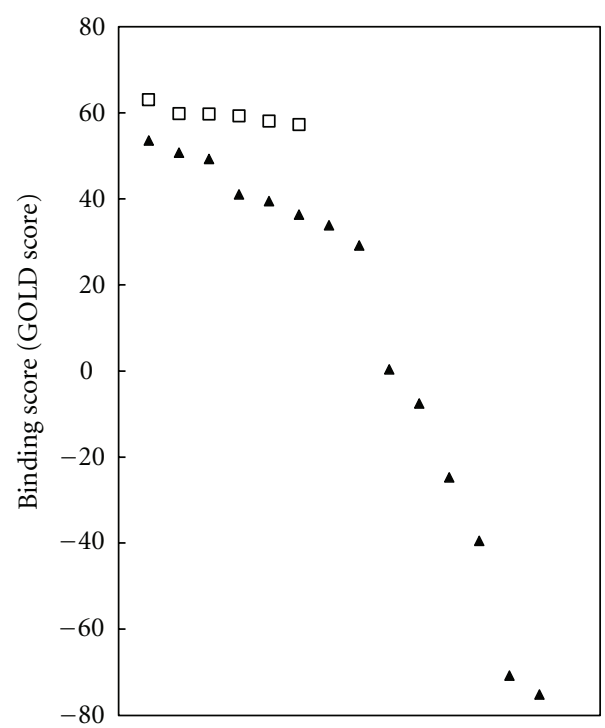

FIGURE 5: Ligand-receptor docking score (GOLD score) when docking with known agonists and antagonists of BLT1. Open squares show docking scores of known BLT1 agonists. Filled triangles show docking scores of known BLT1 antagonists. PubChem ID of BLT1 agonists (descending order): 5280492, 5280745, 5283156, 5280876, 5283129, 5280877. PubChem ID of BLT1 antagonists (descending order): 159476, 3081307, 6444688, 6439064, 6449854, 6540750, 132425, 6439436, 115100, 6442838, 204055, 177941, $196905,192617$.

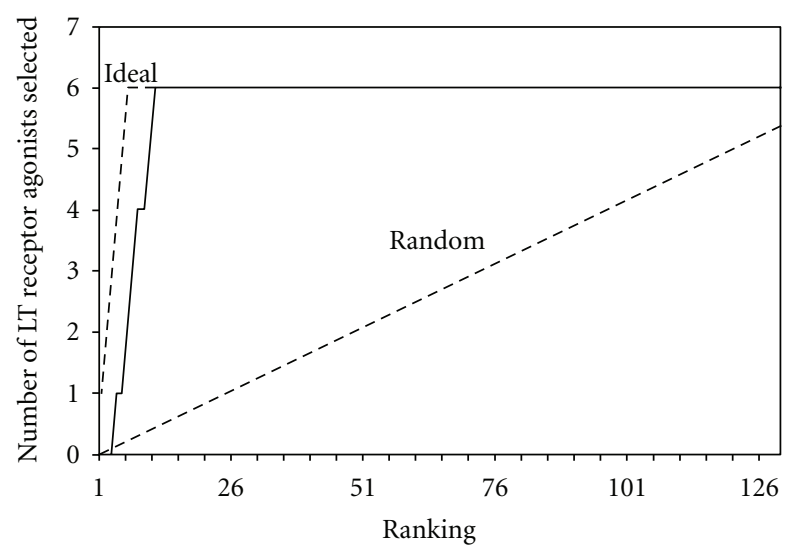

FIGURE 6: Enrichment curve for various GPCR ligands docked into the predicted active-state BLT1 structure.

\section{Discussion and Conclusions}

With no crystal structures of active-state GPCRs other than mutant rhodopsin [34], it is indispensable to develop a practical method to predict 3D structures of activated GPCRs from their sequence information. In this study, we proposed a method for finding one of the best structures of an activated GPCR at a level of accuracy acceptable to virtual screening. The method was validated using human leukotriene B4 receptor BLT1 as a sample GPCR. Our study demonstrated that the proposed method including homology modeling, 
TABLE 1: Ligands used for evaluation.

\begin{tabular}{|c|c|c|c|c|c|}
\hline No. & PubChem ID & Receptor name & Ligand & GLIDA ID & Molecular weight \\
\hline 1 & 5280492 & Leukotriene receptor & Agonist & L000354 & 336 \\
\hline 2 & 5280745 & Leukotriene receptor & Agonist & L000056 & 352 \\
\hline 3 & 5283156 & Leukotriene receptor & Agonist & L000050 & 320 \\
\hline 4 & 5280876 & Leukotriene receptor & Agonist & L000049 & 334 \\
\hline 5 & 5283129 & Leukotriene receptor & Agonist & & 336 \\
\hline 6 & 5280877 & Leukotriene receptor & Agonist & L000055 & 366 \\
\hline 7 & 159476 & Leukotriene receptor & Antagonist & L001349 & 304 \\
\hline 8 & 3081307 & Leukotriene receptor & Antagonist & L011298 & 414 \\
\hline 9 & 6444688 & Leukotriene receptor & Antagonist & L002037 & 502 \\
\hline 10 & 6439064 & Leukotriene receptor & Antagonist & L002263 & 360 \\
\hline 11 & 6449854 & Leukotriene receptor & Antagonist & L000610 & 361 \\
\hline 12 & 6540750 & Leukotriene receptor & Antagonist & L015882 & 346 \\
\hline 13 & 132425 & Leukotriene receptor & Antagonist & L006854 & 358 \\
\hline 14 & 6439436 & Leukotriene receptor & Antagonist & L009515 & 536 \\
\hline 15 & 115100 & Leukotriene receptor & Antagonist & L000493 & 481 \\
\hline 16 & 6442838 & Leukotriene receptor & Antagonist & L001488 & 455 \\
\hline 17 & 204055 & Leukotriene receptor & Antagonist & L006005 & 537 \\
\hline 18 & 177941 & Leukotriene receptor & Antagonist & L001468 & 544 \\
\hline 19 & 196905 & Leukotriene receptor & Antagonist & L006944 & 539 \\
\hline 20 & 192617 & Leukotriene receptor & Antagonist & L011726 & 600 \\
\hline 21 & 68555 & Dopamine receptor & Agonist & L013392 & 307 \\
\hline 22 & 55483 & Dopamine receptor & Agonist & L013398 & 356 \\
\hline 23 & 5760 & Dopamine receptor & Agonist & L000836 & 303 \\
\hline 24 & 3341 & Dopamine receptor & Agonist & L000254 & 306 \\
\hline 25 & 5281878 & Dopamine receptor & Antagonist & L000188 & 434 \\
\hline 26 & 4748 & Dopamine receptor & Antagonist & L000919 & 403 \\
\hline 27 & 28864 & Dopamine receptor & Antagonist & L000349 & 338 \\
\hline 28 & 5265 & Dopamine receptor & Antagonist & L000569 & 395 \\
\hline 29 & 2818 & Dopamine receptor & Antagonist & L000195 & 327 \\
\hline 30 & 5074 & Dopamine receptor & Antagonist & L001003 & 477 \\
\hline 31 & 4038180 & Adrenoceptors & Agonist & L000090 & 309 \\
\hline 32 & 3251 & Adrenoceptors & Agonist & L000768 & 581 \\
\hline 33 & 13109 & Adrenoceptors & Agonist & L000895 & 312 \\
\hline 34 & 2419 & Adrenoceptors & Antagonist & L000137 & 385 \\
\hline 35 & 3372 & Adrenoceptors & Antagonist & L000257 & 437 \\
\hline 36 & 5640 & Adrenoceptors & Antagonist & L000080 & 401 \\
\hline 37 & 47811 & Adrenoceptors & Antagonist & L000474 & 314 \\
\hline 38 & 2369 & Adrenoceptors & Antagonist & L000125 & 307 \\
\hline 39 & 2790 & Histamine receptor & Antagonist & L000192 & 308 \\
\hline 40 & 55482 & Histamine receptor & Antagonist & L000593 & 441 \\
\hline 41 & 41376 & Histamine receptor & Antagonist & L000313 & 321 \\
\hline 42 & 3219 & Histamine receptor & Antagonist & L001093 & 302 \\
\hline 43 & 5533 & Histamine receptor & Antagonist & L000771 & 371 \\
\hline 44 & 65895 & Histamine receptor & Antagonist & L000115 & 380 \\
\hline 45 & 3947 & Histamine receptor & Antagonist & L000944 & 418 \\
\hline 46 & 2678 & Histamine receptor & Antagonist & L000655 & 388 \\
\hline 47 & 3957 & Histamine receptor & Antagonist & L000667 & 382 \\
\hline 48 & 2342 & Histamine receptor & Antagonist & L000844 & 404 \\
\hline 49 & 4830 & Histamine receptor & Antagonist & L000727 & 375 \\
\hline 50 & 475096 & Histamine receptor & Antagonist & L000558 & 440 \\
\hline
\end{tabular}


Table 1: Continued.

\begin{tabular}{|c|c|c|c|c|c|}
\hline No. & PubChem ID & Receptor name & Ligand & GLIDA ID & Molecular weight \\
\hline 51 & 4940 & Histamine receptor & Antagonist & L000807 & 340 \\
\hline 52 & 4066 & Histamine receptor & Antagonist & L000704 & 322 \\
\hline 53 & 3348 & Histamine receptor & Antagonist & L000869 & 501 \\
\hline 54 & 124488 & Histamine receptor & Antagonist & L001444 & 608 \\
\hline 55 & 5282450 & Histamine receptor & Antagonist & L001378 & 392 \\
\hline 56 & 132059 & Serotonin receptor & Agonist & L000336 & 411 \\
\hline 57 & 127728 & Serotonin receptor & Agonist & L000873 & 465 \\
\hline 58 & 197706 & Serotonin receptor & Agonist & L000746 & 403 \\
\hline 59 & 57347 & Serotonin receptor & Agonist & L000797 & 415 \\
\hline 60 & 219050 & Serotonin receptor & Agonist & L001062 & 346 \\
\hline 61 & 3408722 & Serotonin receptor & Agonist & L000428 & 365 \\
\hline 62 & 4440 & Serotonin receptor & Agonist & L000432 & 335 \\
\hline 63 & 56971 & Serotonin receptor & Agonist & L000682 & 401 \\
\hline 64 & 91273 & Serotonin receptor & Agonist & L000863 & 383 \\
\hline 65 & 71351 & Serotonin receptor & Agonist & L001106 & 420 \\
\hline 66 & 5311258 & Serotonin receptor & Agonist & L000364 & 351 \\
\hline 67 & 5761 & Serotonin receptor & Agonist & L000352 & 323 \\
\hline 68 & 5311097 & Serotonin receptor & Agonist & L000365 & 351 \\
\hline 69 & 37816 & Serotonin receptor & Agonist & L000794 & 340 \\
\hline 70 & 3292447 & Serotonin receptor & Agonist & L001346 & 486 \\
\hline 71 & 16362 & Serotonin receptor & Antagonist & L000494 & 461 \\
\hline 72 & 3559 & Serotonin receptor & Antagonist & L000288 & 375 \\
\hline 73 & 55216 & Serotonin receptor & Antagonist & L000503 & 346 \\
\hline 74 & 4431 & Serotonin receptor & Antagonist & L000685 & 393 \\
\hline 75 & 5073 & Serotonin receptor & Antagonist & L000510 & 410 \\
\hline 76 & 2726 & Serotonin receptor & Antagonist & L000182 & 318 \\
\hline 77 & 55752 & Serotonin receptor & Antagonist & L000720 & 440 \\
\hline 78 & 5684 & Serotonin receptor & Antagonist & L000012 & 422 \\
\hline 79 & 28693 & Serotonin receptor & Antagonist & L000396 & 403 \\
\hline 80 & 4106 & Serotonin receptor & Antagonist & L000397 & 356 \\
\hline 81 & 68848 & Serotonin receptor & Antagonist & L000394 & 362 \\
\hline 82 & 37459 & Serotonin receptor & Antagonist & L000016 & 361 \\
\hline 83 & 107780 & Serotonin receptor & Antagonist & L000275 & 497 \\
\hline 84 & 3378093 & Serotonin receptor & Antagonist & L000538 & 520 \\
\hline 85 & 1229 & Serotonin receptor & Agonist & L000011 & 321 \\
\hline 86 & 3654103 & Serotonin receptor & Agonist & L000142 & 406 \\
\hline 87 & 4585 & Serotonin receptor & Antagonist & L000455 & 312 \\
\hline 88 & 133083 & Angiotensin receptor & Antagonist & L008043 & 490 \\
\hline 89 & 114899 & Angiotensin receptor & Antagonist & L000467 & 480 \\
\hline 90 & 3749 & Angiotensin receptor & Antagonist & L000319 & 428 \\
\hline 91 & 60921 & Angiotensin receptor & Antagonist & L000533 & 610 \\
\hline 92 & 2541 & Angiotensin receptor & Antagonist & L000156 & 440 \\
\hline 93 & 5281037 & Angiotensin receptor & Antagonist & L000248 & 424 \\
\hline 94 & 60846 & Angiotensin receptor & Antagonist & L000621 & 435 \\
\hline 95 & 5833 & Angiotensin receptor & Antagonist & L001221 & 416 \\
\hline 96 & 472880 & Follicle stimulating hormone & Agonist & L000979 & 352 \\
\hline 97 & 125672 & Melanocortin hormone & Agonist & L000639 & 342 \\
\hline 98 & 6434259 & Prostanoid receptor & Agonist & L000194 & 424 \\
\hline 99 & 6436393 & Prostanoid receptor & Agonist & L000160 & 350 \\
\hline 100 & 5311027 & Prostanoid receptor & Agonist & L001211 & 415 \\
\hline
\end{tabular}


TABle 1: Continued.

\begin{tabular}{|c|c|c|c|c|c|}
\hline No. & PubChem ID & Receptor name & Ligand & GLIDA ID & Molecular weight \\
\hline 101 & 5311503 & Prostanoid receptor & Agonist & L000634 & 384 \\
\hline 102 & 119304 & Prostanoid receptor & Agonist & L000151 & 368 \\
\hline 103 & 5311493 & Prostanoid receptor & Agonist & L024041 & 350 \\
\hline 104 & 6433212 & Prostanoid receptor & Agonist & L000478 & 354 \\
\hline 105 & 6439022 & Prostanoid receptor & Agonist & L000570 & 362 \\
\hline 106 & 122021 & Prostanoid receptor & Antagonist & L000153 & 459 \\
\hline 107 & 160 & Prostanoid receptor & Antagonist & L000705 & 354 \\
\hline 108 & 5311213 & Prostanoid receptor & Antagonist & L024043 & 442 \\
\hline 109 & 50294 & Prostanoid receptor & Antagonist & L001326 & 371 \\
\hline 110 & 132836 & Prostanoid receptor & Agonist & L000098 & 388 \\
\hline 111 & 5311035 & Prostanoid receptor & Agonist & L000150 & 408 \\
\hline 112 & 164437 & Adenosine receptor & Agonist & L000290 & 388 \\
\hline 113 & 104795 & Adenosine receptor & Agonist & L000437 & 308 \\
\hline 114 & 164305 & Adenosine receptor & Agonist & L001390 & 363 \\
\hline 115 & 93205 & Adenosine receptor & Agonist & L000708 & 385 \\
\hline 116 & 123807 & Adenosine receptor & Agonist & L000165 & 369 \\
\hline 117 & 3086599 & Adenosine receptor & Agonist & L001283 & 499 \\
\hline 118 & 5311506 & Adenosine receptor & Antagonist & L000893 & 337 \\
\hline 119 & 1329 & Adenosine receptor & Antagonist & L000233 & 304 \\
\hline 120 & 393595 & Adenosine receptor & Antagonist & L000629 & 403 \\
\hline 121 & 176408 & Adenosine receptor & Antagonist & L000548 & 345 \\
\hline 122 & 5697 & Adenosine receptor & Antagonist & L000963 & 428 \\
\hline 123 & 64627 & Adenosine receptor & Antagonist & L001406 & 356 \\
\hline 124 & 1970 & Adenosine receptor & Agonist & L001216 & 399 \\
\hline 125 & 9576912 & Adenosine receptor & Agonist & L001516 & 393 \\
\hline 126 & 122246 & Adenosine receptor & Agonist & L000951 & 521 \\
\hline 127 & 5287468 & Adenosine receptor & Agonist & L000058 & 526 \\
\hline 128 & 123739 & Adenosine receptor & Antagonist & L001123 & 386 \\
\hline 129 & 16078 & Cannabinoid receptor & Agonist & L000023 & 314 \\
\hline 130 & 5281969 & Cannabinoid receptor & Agonist & L000111 & 347 \\
\hline 131 & 5689 & Cannabinoid receptor & Agonist & L000626 & 426 \\
\hline 132 & 5488671 & Cannabinoid receptor & Agonist & L000222 & 395 \\
\hline 133 & 104895 & Cannabinoid receptor & Agonist & L000004 & 376 \\
\hline 134 & 39860 & Cannabinoid receptor & Agonist & L000994 & 372 \\
\hline 135 & 5311257 & Cannabinoid receptor & Antagonist & L000363 & 383 \\
\hline 136 & 4172142 & Melatonin receptor & Agonist & L000306 & 348 \\
\hline 137 & 4004 & Melatonin receptor & Antagonist & L001138 & 330 \\
\hline 138 & 5311198 & Melatonin receptor & Antagonist & L000326 & 376 \\
\hline 139 & 6024 & Calcitonin receptor & Agonist & L000905 & 396 \\
\hline 140 & 91498 & Calcitonin receptor & Agonist & L024117 & 421 \\
\hline 141 & 446284 & Metabotropic glutamate group I & Agonist & L001256 & 302 \\
\hline 142 & 6324636 & Metabotropic glutamate group I & Antagonist & L000366 & 323 \\
\hline 143 & 5311262 & Metabotropic glutamate group I & Antagonist & L000372 & 383 \\
\hline 144 & 123885 & GABA-B subtype 1 & Antagonist & L000172 & 401 \\
\hline
\end{tabular}

MD simulations, and evolutionary computing was able to provide the appropriate $3 \mathrm{D}$ structure of the activated leukotriene receptor to specifically dock with its agonists.

The first remarkable feature of this study is that the proposed method models GPCR thermal fluctuations on multiple time scales: the short-term heat fluctuation on the picosecond or nanosecond time scale and the rotational motions of TM helices on a longer time scale. This is a kind of coarse approximation compared with other methods like rigorous long-term MD simulations. However, the proposed method provides a significant reduction in computational cost when predicting the 3D structure of GPCRs and finally 
leads us to obtain the structure on common personal computers.

The second feature is that a machine learning technique including evolutionary computation and modularized ligand-binding simulations is employed for the structural prediction. Though to date, there have been methods using GAs to generate various conformations of ligands to explore a most suitable conformation in ligand-receptor docking [36], our method is the first approach using a GA employing a modularized ligand-binding simulation to find a best conformation on the receptor side.

However, some improvements might remain in the evaluation function of our evolutionary computing. Essentially, a scoring system capable of closely reflecting the binding-free energy would be desirable for the conformation evaluation. In our method, the GOLD scores produced by the GOLD software are directly used for the evaluation function. Although the GOLD scores are related with the values of the binding-free energy, they are not identical. We should accordingly investigate this issue in the future.

In addition, it should be noted that our method has a tendency to predict the central part of the 7-TM helical bundle, a spacious ligand binding site, with good quality. That is, small deviations would be observed in a spacious binding pocket area, whereas larger deviations would be observed in the distal section of the helices and even larger in the loop regions. This is because the evaluation function in evolutionary computing is naturally based on the ligandbinding score, closely related with the binding free energy. Hence, our method may predict another structure having energetically highly evaluated conformations of seven helices and the loops as a best structure if another search is performed. However, the structure of the spacious ligandbinding site would emerge again. We originally have the purpose of using the predicted 3D structure for virtual screening of candidate chemicals. Our method is able to provide $3 \mathrm{D}$ structure accurately enough for this purpose as shown in the case of the leukotriene receptor, since the structural information of the spacious binding pocket area is the most important for virtual screening.

We anticipate that parallelization of the proposed algorithm would contribute to an improvement in computation time. Future study designs will incorporate such. Furthermore, we plan to use GPCRs other than the leukotriene receptor to test the applicability and robustness of our search method. For such investigations, it would be important to carefully select an appropriate target GPCR. Based on sequence similarity within the seven TMs, GPCRs can be grouped into six families: the rhodopsin family $A$, the secretin family $B$, the glutamate receptor family $C$, the fungal pheromone family D, the cAMP receptor family $\mathrm{E}$, and the frizzled/smoothened receptor family $F$ [37]. In general, GPCRs belonging to class A fit the prediction, because all known GPCRs used as templates in homology modeling are classified into the class A. In contrast, other GPCRs having low amino-acid homology would need extra processing at the preliminary stage of homology modeling.

\section{Acknowledgment}

This work was in part supported by the Grant-in-Aid for Scientific Research from the Ministry of Education, Culture, Sports, Science, and Technology of Japan.

\section{References}

[1] V. L. Lowes, N. Y. Ip, and Y. H. Wong, "Integration of signals from receptor tyrosine kinases and $G$ proteincoupled receptors," NeuroSignals, vol. 11, no. 1, pp. 5-19, 2002.

[2] K. J. Miller, B. J. Murphy, and M. A. Pelleymounter, "Central G-protein coupled receptors (GPCR)s as molecular targets for the treatment of obesity: assets, liabilities and development status," Current Drug Targets, vol. 3, no. 5, pp. 357-377, 2004.

[3] S. L. Parker, M. S. Parker, R. Sah, and F. Sallee, "Angiogenesis and rhodopsin-like receptors: a role for N-terminal acidic residues?" Biochemical and Biophysical Research Communications, vol. 335, no. 4, pp. 983-992, 2005.

[4] G. V. Rayasam, V. K. Tulasi, J. A. Davis, and V. S. Bansal, "Fatty acid receptors as new therapeutic targets for diabetes," Expert Opinion on Therapeutic Targets, vol. 11, no. 5, pp. 661-671, 2007.

[5] J. Drews, "Drug discovery: a historical perspective," Science, vol. 287, no. 5460, pp. 1960-1964, 2000.

[6] D. K. Vassilatis, J. G. Hohmann, H. Zeng et al., "The G protein-coupled receptor repertoires of human and mouse," Proceedings of the National Academy of Sciences of the United States of America, vol. 100, no. 8, pp. 4903-4908, 2003.

[7] K. Lundstrom, "Structural genomics and drug discovery: molecular pharmacology," Journal of Cellular and Molecular Medicine, vol. 11, no. 2, pp. 224-238, 2007.

[8] K. Palczewski, T. Kumasaka, T. Hori et al., "Crystal structure of rhodopsin: a G protein-coupled receptor," Science, vol. 289, no. 5480, pp. 739-745, 2000.

[9] V. Cherezov, D. M. Rosenbaum, M. A. Hanson et al., "Highresolution crystal structure of an engineered human $\beta 2$ adrenergic G protein-coupled receptor," Science, vol. 318, no. 5854, pp. 1258-1265, 2007.

[10] D. M. Rosenbaum, V. Cherezov, M. A. Hanson et al., "GPCR engineering yields high-resolution structural insights into $\beta 2$ adrenergic receptor function," Science, vol. 318, no. 5854, pp. 1266-1273, 2007.

[11] T. Warne, M. J. Serrano-Vega, J. G. Baker et al., "Structure of a $\beta 1$-adrenergic G-protein-coupled receptor," Nature, vol. 454, no. 7203, pp. 486-491, 2008.

[12] V. P. Jaakola, M. T. Griffith, M. A. Hanson et al., "The 2.6 angstrom crystal structure of a human A2A adenosine receptor bound to an antagonist," Science, vol. 322, no. 5905, pp. 1211-1217, 2008.

[13] H. Wu, D. Wacker, M. Mileni et al., "Structure of the human $\kappa$ opioid receptor in complex with JDTic," Nature, vol. 485, pp. 327-332, 2012.

[14] A. Manglik, A. C. Kruse, T. S. Kobilka et al., "Crystal structure of the $\mu$-opioid receptor bound to a morphinan antagonist," Nature, vol. 485, pp. 321-326, 2012.

[15] J. Moult, K. Fidelis, A. Kryshtafovych, B. Rost, and A. Tramontano, "Critical assessment of methods of protein structure prediction-Round VIII," Proteins, vol. 77, no. 9, pp. 1-4, 2009.

[16] M. Michino, E. Abola, C. L. Brooks III, J. S. Dixon, J. Moult, 
and R. C. Stevens, "Community-wide assessment of GPCR structure modelling and ligand docking: GPCR Dock 2008," Nature Reviews Drug Discovery, vol. 8, no. 6, pp. 455-463, 2009.

[17] M. R. Whorton, M. P. Bokoch, S. G. F. Rasmussen et al., "A monomeric G protein-coupled receptor isolated in a highdensity lipoprotein particle efficiently activates its G protein," Proceedings of the National Academy of Sciences of the United States of America, vol. 104, no. 18, pp. 7682-7687, 2007.

[18] C. Altenbach, A. K. Kusnetzow, O. P. Ernst, K. P. Hofmann, and W. L. Hubbell, "High-resolution distance mapping in rhodopsin reveals the pattern of helix movement due to activation," Proceedings of the National Academy of Sciences of the United States of America, vol. 105, no. 21, pp. 7439-7444, 2008.

[19] F. Fanelli and D. Dell'Orco, "Rhodopsin activation follows precoupling with transducin: inferences from computational analysis," Biochemistry, vol. 44, no. 45, pp. 14695-14700, 2005.

[20] B. Isin, A. J. Rader, H. K. Dhiman, J. Klein-Seetharaman, and I. Bahar, "Predisposition of the dark state of rhodopsin to functional changes in structure," Proteins: Structure, Function and Genetics, vol. 65, no. 4, pp. 970-983, 2006.

[21] M. Y. Niv, L. Skrabanek, M. Filizola, and H. Weinstein, "Modeling activated states of GPCRs: the rhodopsin template," Journal of Computer-Aided Molecular Design, vol. 20, no. 7-8, pp. 437-448, 2006.

[22] P. R. Gouldson, N. J. Kidley, R. P. Bywater et al., "Toward the active conformations of rhodopsin and the $\beta$-adrenergic receptor," Proteins: Structure, Function and Genetics, vol. 56, no. 1, pp. 67-84, 2004.

[23] H. Saitô, Y. Kawase, A. Kira et al., "Surface and dynamic structures of bacteriorhodopsin in a 2D crystal a distorted or disrupted lattice, as revealed by site-directed solid-state $13 \mathrm{C}$ NMR," Photochemistry and Photobiology, vol. 83, no. 2, pp. 253-262, 2007.

[24] Z. L. Lu and E. C. Hulme, "A network of conserved intramolecular contacts defines the off-state of the transmembrane switch mechanism in a seven-transmembrane receptor," Journal of Biological Chemistry, vol. 275, no. 8, pp. 5682-5686, 2000.

[25] L. J. Eshelman and J. D. Schaffer, "Real-coded genetic algorithms and interval-schemata," Foundations of Genetic Algorithms, vol. 2, pp. 187-202, 1993.

[26] I. Ono, H. Sato, and S. Kobayashi, "A real-coded genetic algorithm for function optimization using the unimodal normal distribution crossover," The Japanese Society for Artificial Intelligence, vol. 14, pp. 1146-1155, 1999.

[27] H. Sato, I. Ono, and S. Kobayashi, "A new generation alternation model of genetic algorithms and its assessment," The Japanese Society for Artificial Intelligence, vol. 12, pp. 734744, 1997.

[28] J. D. Thompson, T. J. Gibson, F. Plewniak, F. Jeanmougin, and D. G. Higgins, "The CLUSTAL X windows interface: flexible strategies for multiple sequence alignment aided by quality analysis tools," Nucleic Acids Research, vol. 25, no. 24, pp. 4876-4882, 1997.

[29] U. Pieper, N. Eswar, B. M. Webb et al., "MODBASE, a database of annotated comparative protein structure models and associated resources," Nucleic Acids Research, vol. 37, no. 1, pp. D347-D354, 2009.

[30] A. Sali, "ModPipe: a software to calculate protein structure models," 2012, http://salilab.org/modpipe/.

[31] J. W. Ponder, “Tinker: a software for molecular design,” 2012, http://dasher.wustl.edu/tinker/.

[32] A. Sabirsh, R. P. Bywater, J. Bristulf, C. Owman, and J. Z. Haeggström, "Residues from transmembrane helices 3 and 5 participate in leukotriene B4 binding to BLT1," Biochemistry, vol. 45, no. 18, pp. 5733-5744, 2006.

[33] M. L. Verdonk, J. C. Cole, M. J. Hartshorn, C. W. Murray, and R. D. Taylor, "Improved protein-ligand docking using GOLD," Proteins: Structure, Function and Genetics, vol. 52, no. 4, pp. 609-623, 2003.

[34] J. Standfuss, P. C. Edwards, A. D’Antona et al., "The structural basis of agonist-induced activation in constitutively active rhodopsin," Nature, vol. 471, no. 7340, pp. 656-660, 2011.

[35] C. Bissantz, P. Bernard, M. Hibert, and D. Rognan, "Proteinbased virtual screening of chemical databases. II. Are homology models of G-protein coupled receptors suitable targets?" Proteins: Structure, Function and Genetics, vol. 50, no. 1, pp. 5-25, 2003.

[36] C. M. Oshiro, I. D. Kuntz, and J. S. Dixon, "Flexible ligand docking using a genetic algorithm," Journal of Computer-Aided Molecular Design, vol. 9, no. 2, pp. 113-130, 1995.

[37] J. C. Mobarec and M. Filizola, "Advances in the development and application of computational methodologies for structural modeling of G-protein-coupled receptors," Expert Opinion on Drug Discovery, vol. 3, no. 3, pp. 343-355, 2008. 

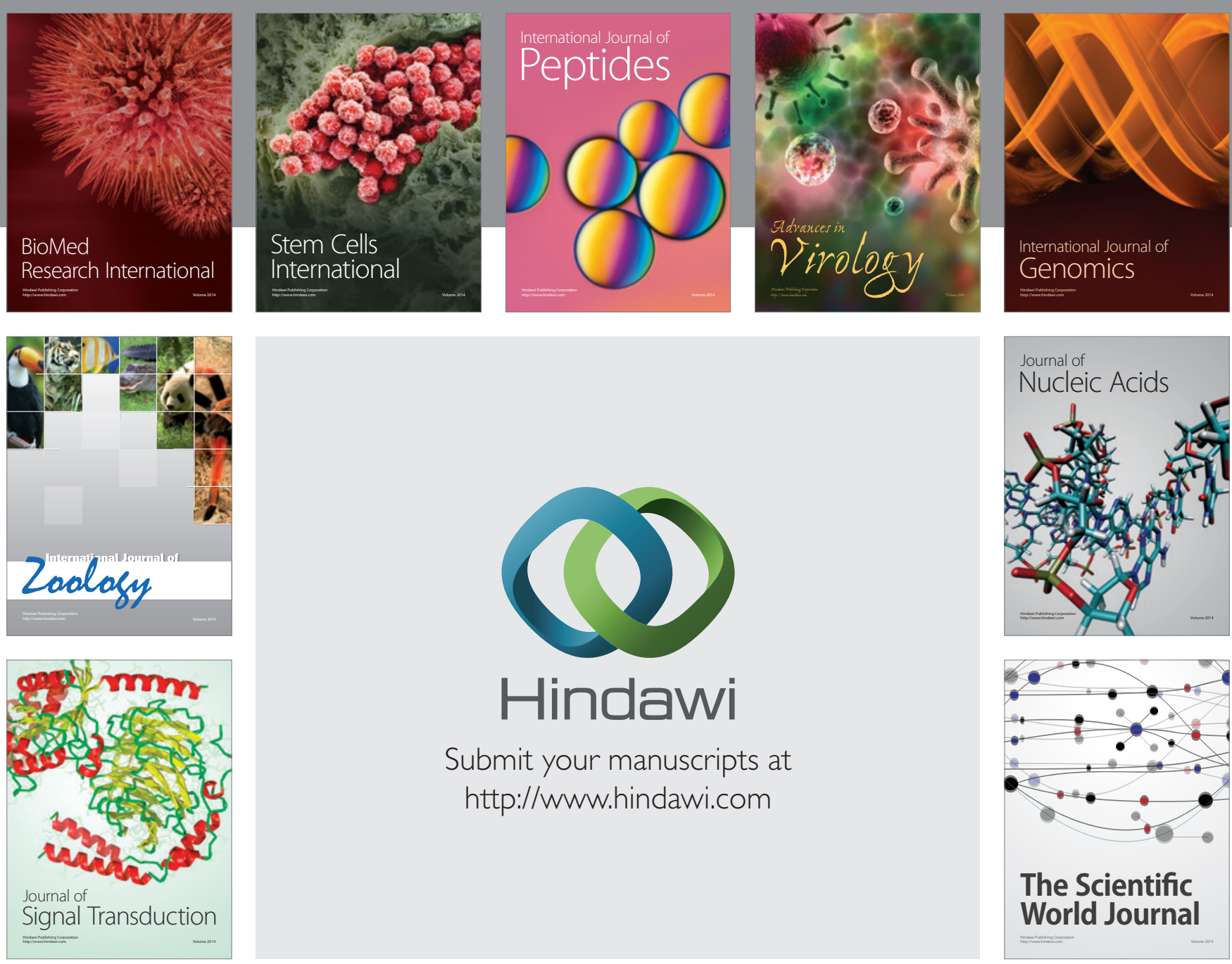

Submit your manuscripts at

http://www.hindawi.com
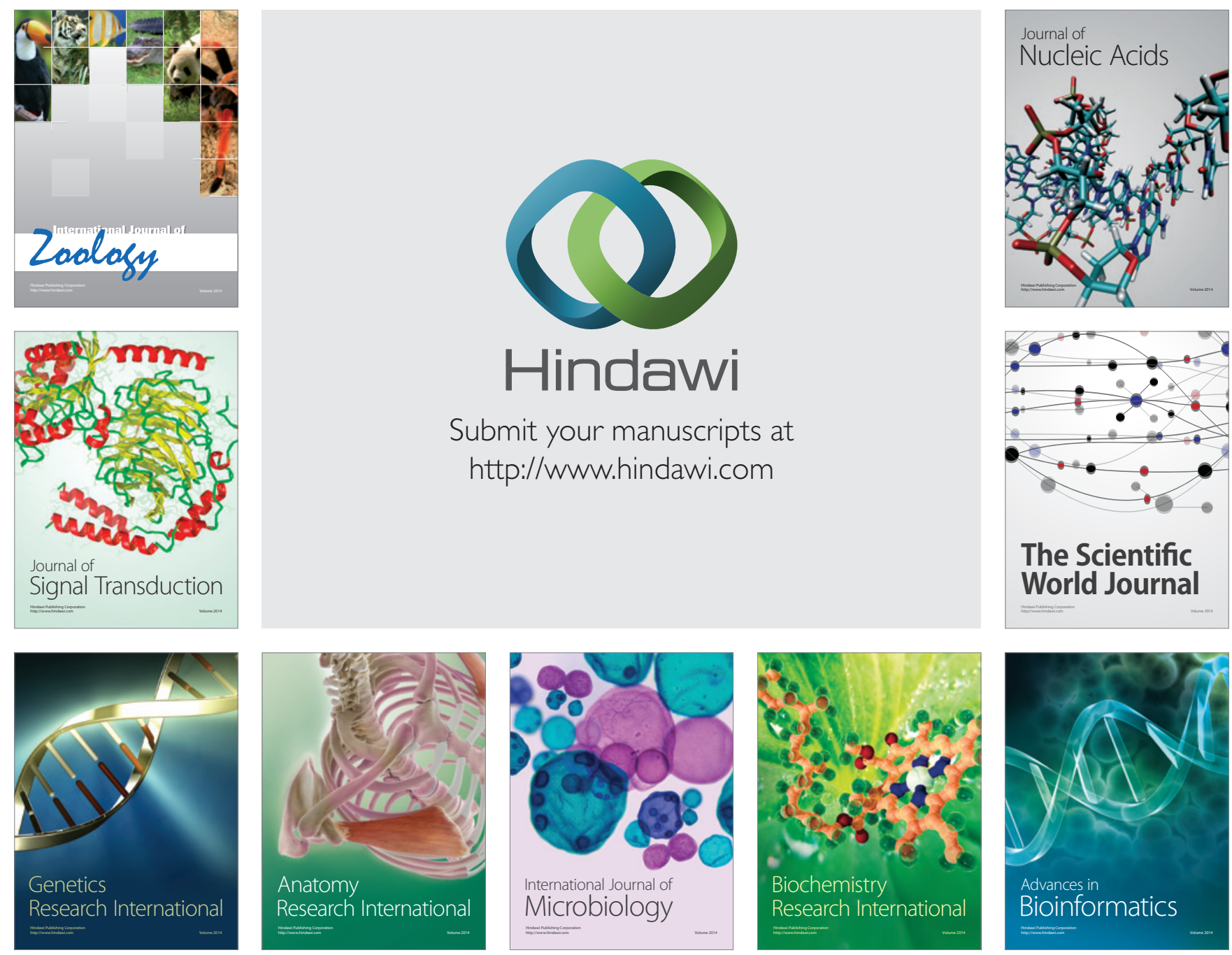

The Scientific World Journal
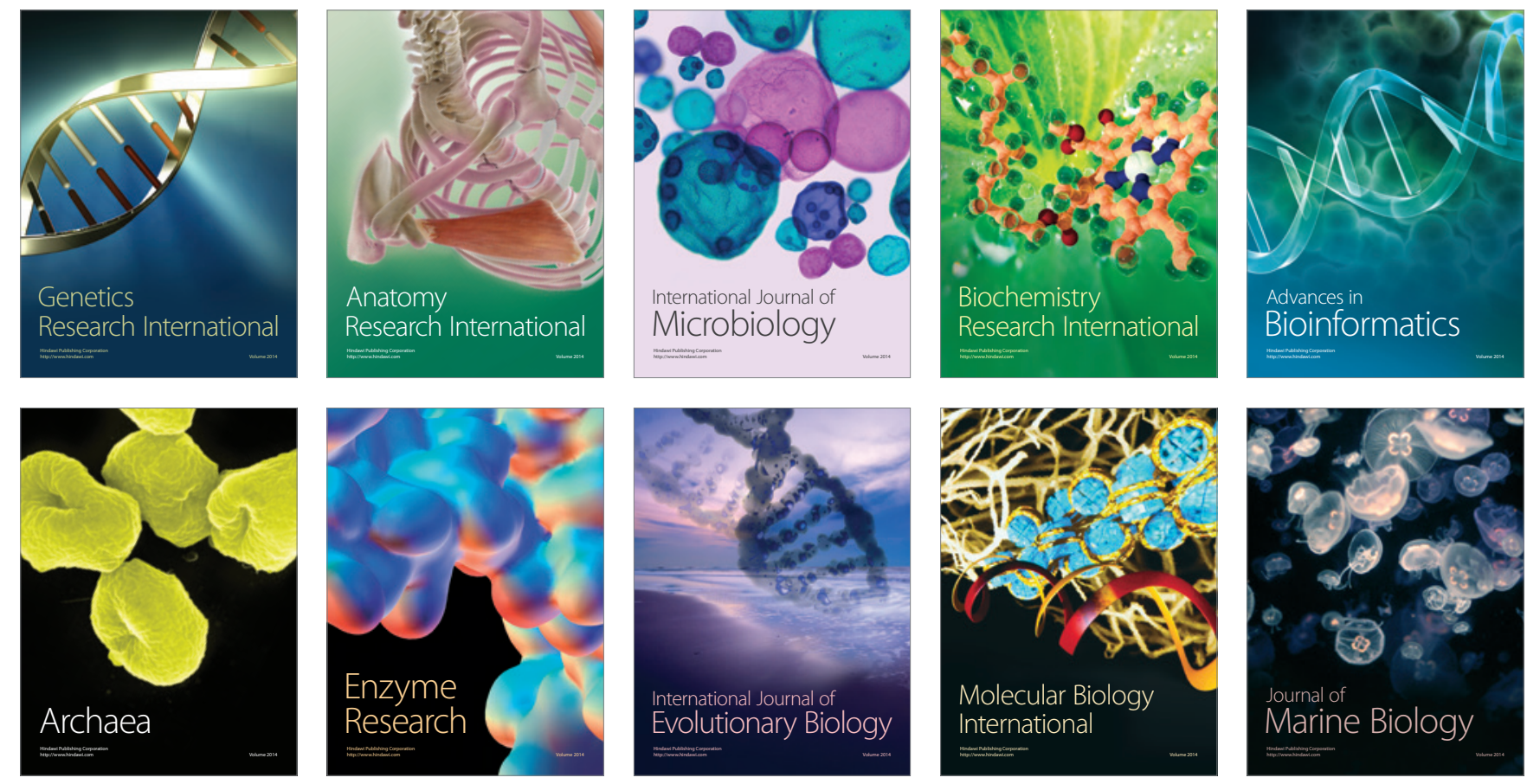